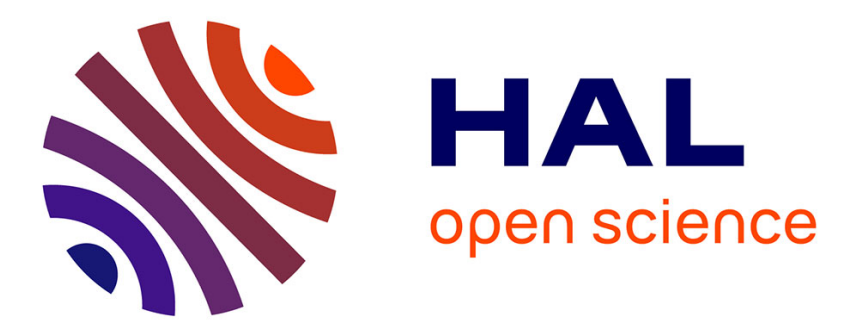

\title{
Mesure des émissivités et des réflectivités monochromatiques directionnelles des matériaux opaques
}

\author{
M. Huetz-Aubert, J.F. Sacadura
}

\section{- To cite this version:}

M. Huetz-Aubert, J.F. Sacadura. Mesure des émissivités et des réflectivités monochromatiques directionnelles des matériaux opaques. Revue de Physique Appliquée, 1982, 17 (4), pp.251-260. 10.1051/rphysap:01982001704025100 . jpa-00244994

\section{HAL Id: jpa-00244994 https://hal.science/jpa-00244994}

Submitted on 1 Jan 1982

HAL is a multi-disciplinary open access archive for the deposit and dissemination of scientific research documents, whether they are published or not. The documents may come from teaching and research institutions in France or abroad, or from public or private research centers.
L'archive ouverte pluridisciplinaire HAL, est destinée au dépôt et à la diffusion de documents scientifiques de niveau recherche, publiés ou non, émanant des établissements d'enseignement et de recherche français ou étrangers, des laboratoires publics ou privés. 


\title{
Mesure des émissivités et des réflectivités monochromatiques directionnelles des matériaux opaques
}

\author{
M. Huetz-Aubert (*) et J. F. Sacadura (**) \\ * Groupe de Recherches Thermiques du C.N.R.S. associé à l'Ecole Centrale des Arts et Manufactures, \\ Grande Voie des Vignes, 92290 Châtenay-Malabry, France \\ ** Laboratoire d'Hydraulique et Dynamique des Gaz, Institut National des Sciences Appliquées de Lyon, Bât. 302, \\ 20, avenue Einstein, 69621 Villeurbanne Cedex, France
}

(Reçu le 28 septembre 1981, révisé le 15 décembre 1981, accepté le 22 décembre 1981)

\begin{abstract}
Résumé. - Pour calculer les transferts d'énergie radiative entre matériaux opaques, il est impossible de se contenter de déterminations portant sur des grandeurs totales, qu'elles soient directionnelles ou hémisphériques. La connaissance des émissivités $\varepsilon_{\lambda}^{\prime}$ ou des réflectivités $\rho_{\lambda}^{\prime}{ }^{\circ}=\rho_{\lambda \text { iso }}^{\prime \prime}=1-\varepsilon_{\lambda}^{\prime}$ monochromatiques directionnelles s'avère indispensable. L'exploration d'un large domaine de température $T$ et de longueur d'onde $\lambda$, ainsi que la nécessité de confronter des résultats obtenus par des techniques différentes, impliquent la mise au point de plusieurs montages. Nous décrivons ici deux types d'appareillages exploités dans nos laboratoires pour mesurer respectivement $\varepsilon_{\lambda}^{\prime}$ dans les conditions suivantes : $2<\lambda<15 \mu \mathrm{m}, 500<T<1000 \mathrm{~K}$, et $\rho_{\lambda \text { iso }}^{\prime}$ de $0,3 \mathrm{à} 1 \mu \mathrm{m}$ avec $200<T<1000 \mathrm{~K}$.
\end{abstract}

\begin{abstract}
For the computation of radiative energy transfer between opaque materials, the use of total factors either directional or hemispherical leads to incorrect results. The knowledge of the monochromatic directional emissivity $\varepsilon_{\lambda}^{\prime}$ or reflectivity $\rho_{\lambda}^{\prime \circ}=\rho_{\lambda \text { iso }}^{\prime}=1-\varepsilon_{\lambda}^{\prime}$ is thus necessary. The survey of a wide range of temperature $T$ and wavelength $\lambda$, and the necessity of comparing the results of different techniques implies the realization of various experimental configurations. We shall describe two systems used in our laboratories; the first evaluates $\varepsilon_{\lambda}^{\prime}$ for temperature ranging from 500 to $1000 \mathrm{~K}$ and wavelength, from 2 to $15 \mu \mathrm{m}$; the other measures $\rho_{\lambda \text { iso }}^{\prime}$ in the ranges 250 to $1000 \mathrm{~K}$ and 0.3 to $1 \mu \mathrm{m}$.
\end{abstract}

Introduction. - Les émissivités, absorptivités et réflectivités interviennent dans les calculs d'échanges d'énergie par rayonnement entre matériaux opaques. Lorsqu'on se contente d'un ordre de grandeur, des valeurs moyennées sur l'ensemble des longueurs d'onde $\lambda$ et des directions de propagation $\Delta$ suffisent puisqu'elles sont associées aux hypothèses simplificatrices de surfaces grises, à émission isotrope, à réflexion spéculaire ou isotrope. Mais de plus en plus d'applications exigent un calcul aussi rigoureux que possible. La prise en compte des variations des facteurs radiatifs avec la longueur d'onde $\lambda$, la direction $\Delta$ et la température $T$ du matériau devient alors indispensable; il faut aussi connaître l'influence des états de surface, cette dernière pouvant être plus ou moins rugueuse et parfois contaminée par des couches variées : oxydes, nitrures..

Des méthodes directes, voire in situ, permettant de mesurer les émissivités, absorptivités et réflectivités monochromatiques directionnelles, apparaissent à l'heure actuelle nécessaires dans un large domaine de longueur d'onde et de température $T$, sans exclure celui des basses températures puisque le rayonnement est parfois le seul mode de transfert d'énergie et que, quel que soit $T$, un corps est toujours susceptible d'absorber. Après un examen des différents facteurs mesurables, nous justifierons l'orientation de notre choix vers certains d'entre eux. Les montages, exploités au Groupe de Recherches Thermiques et à l'INSA de Lyon, seront décrits avec une analyse des principales causes d'erreur. A titre d'illustration, quelques résultats seront présentés dans le dernier paragraphe.

\section{Caractéristiques radiatives. Choix du facteur à} mesurer. - 1.1 ReLATIONS ENTRE LES FACTEURS MONOCHROMATIQUES DIRECTIONNELS. BILAN RADIATIF. - L'émissivité monochromatique directionnelle $\varepsilon_{\lambda}^{\prime}$ est le rapport entre le flux $\mathrm{d} \phi_{\lambda e}$, émis par l'élément de surface dS du matériau (Fig. 1), dans une direction $\Delta$ et pour une longueur d'onde $\lambda$, et celui $\mathrm{d} \phi_{\lambda}^{0}$ émis par 


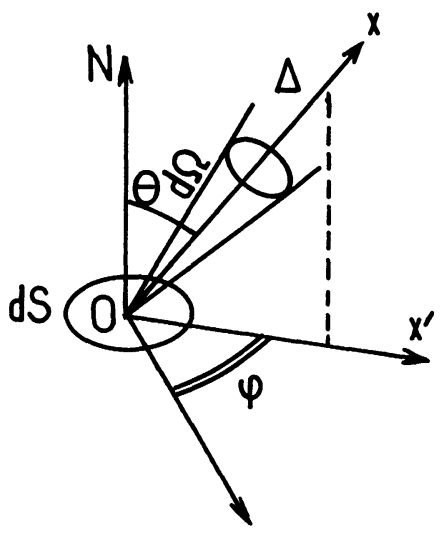

Fig. 1. - Elément de surface $d S$ émettant un flux $\mathrm{d} \phi_{\lambda_{\mathrm{e}}}$ dans la direction $\Delta$ ou recevant un flux $\mathrm{d} \phi_{\lambda \mathrm{i}}$ de cette même direction.

[Elementary surface $d S$ either emitting the flux $d \phi_{\lambda e}$ to the direction $\Delta$ or receiving the flux $\mathrm{d} \phi_{\lambda \mathrm{i}}$ from the same direction.]

le corps noir dans les mêmes conditions; autrement dit :

$$
\varepsilon_{\lambda}^{\prime}(\lambda, \Delta, T)=\frac{\mathrm{d} \phi_{\lambda e}(\lambda, \Delta, T)}{\mathrm{d} \phi_{\lambda}^{0}(\lambda, T)}=\frac{L_{\lambda \mathrm{e}}(\lambda, \Delta, T)}{L_{\lambda}^{0}(\lambda, T)}
$$

$L_{\lambda \mathrm{e}}$ et $L_{\lambda}^{0}$ sont les luminances monochromatiques respectives des flux émis par le matériau et par le corps noir que nous supposerons toujours placés dans le vide ou dans l'air dont l'indice de réfraction peut être posé égal à 1 . La longueur d'onde $\lambda$ est alors liée à la fréquence $v$ du rayonnement par $\lambda=c / v, c$ vitesse des ondes électromagnétiques dans le vide, et, d'après la loi de Planck :

$$
L_{\lambda}^{0}(\lambda, T)=\frac{2 h c^{2} \lambda^{-5}}{\exp (h c / k \lambda T)-1}
$$

$h$ et $k$ constantes respectives de Planck et de Boltzmann, $T$ température absolue en Kelvin.

Etant donné un flux incident $\mathrm{d} \phi_{\lambda \mathrm{i}}$ selon la direction $\Delta$, de luminance $L_{\lambda \mathrm{i}}$, soit $\mathrm{d} \phi_{\lambda_{\mathrm{a}}}$ la part absorbée; l'absorptivité monochromatique directionnelle s'écrit :

$$
\alpha_{\lambda}^{\prime}(\lambda, \Delta, T)=\frac{\mathrm{d} \phi_{\lambda \mathrm{a}}}{\mathrm{d} \phi_{\lambda \mathrm{i}}}
$$

Les différents facteurs de réflexion ont été examinés dans le précédent article [1]. Nous n'envisagerons ici que les deux principaux, c'est-à-dire :

- La réflectivité monochromatique directionnellehémisphérique définie à partir du flux incident $\mathrm{d} \phi_{\lambda_{\mathrm{i}}}$ par :

$$
\rho_{\lambda}^{\prime \circ}(\lambda, \Delta, T)=\frac{\mathrm{d} \phi_{\lambda \mathrm{r}}}{\mathrm{d} \phi_{\lambda \mathrm{i}}}
$$

où $\mathrm{d} \phi_{\lambda r}$ représente le flux réfléchi dans toutes les directions.
- Inversement, lorsqu'un élément dS reçoit un flux de luminance moyenne $\bar{L}_{\lambda \mathrm{i}}$ provenant du demiespace entourant le matériau, la réflectivité monochromatique hémisphérique-directionnelle $\dot{\rho}_{\lambda}^{\prime \prime}$ exprime le rapport entre la luminance du flux réfléchi selon $\Delta$ et $\bar{L}_{\lambda \mathrm{i}}$. Ce facteur est peu employé, car il dépend de la distribution spatiale du flux incident [2]; il ne devient intéressant que si celui-ci est isotrope avec $\bar{L}_{\lambda \mathrm{i}}^{\prime}=L_{\lambda \mathrm{i}}$; nous utiliserons alors la notation $\rho_{\lambda \text { iso. }}^{\mathrm{\alpha}}$.

La conservation de l'énergie, entre le flux incident $\mathrm{d} \phi_{\lambda \mathrm{i}}$ d'une part, les flux réfléchi $\mathrm{d} \phi_{\lambda \mathrm{r}}$ et absorbé $\mathrm{d} \phi_{\lambda \mathrm{a}}$ d'autre part, conduit à une relation très simple entre $\alpha_{\lambda}^{\prime}$ et $\rho_{\lambda}^{\prime O}$ :

$$
\alpha_{\lambda}^{\prime}+\rho_{\lambda}^{\prime \circ}=1 .
$$

On peut également montrer [3] qu'une relation analogue existe entre $\varepsilon_{\lambda}^{\prime}$ et $\rho_{\lambda \text { iso }}^{\prime \prime}$ :

$$
\varepsilon_{\lambda}^{\prime}+\rho_{\lambda \text { iso }}^{\circ \prime}=1 .
$$

Par ailleurs, l'utilisation du principe de réciprocité de Helmholtz [4], dont la validité n'a pas été mise en cause jusqu'ici, entraîne l'égalité :

$$
\rho_{\lambda \text { iso }}^{\prime \prime}=\rho_{\lambda}^{\prime \prime}
$$

et, par conséquent, la loi de Kirchhoff ou de Draper :

$$
\varepsilon_{\lambda}^{\prime}=\alpha_{\lambda}^{\prime} .
$$

Les flux surfaciques, émis $\varphi_{\mathrm{e}}$, absorbé $\varphi_{\mathrm{a}}$ ou réfléchi $\varphi_{\mathrm{r}}$ par chaque élément dS du matériau, s'expriment immédiatement à l'aide des facteurs définis ci-dessus; c'est ainsi que :

$$
\varphi_{\mathrm{e}}=\int_{0} \int_{0}^{\infty} \varepsilon_{\lambda}^{\prime} L_{\lambda}^{0}(T) \cos \theta \mathrm{d} \Omega \mathrm{d} \lambda
$$

et que, compte tenu de (8) :

$$
\varphi_{\mathrm{a}}=\int_{\Delta} \int_{0}^{\infty} \varepsilon_{\lambda}^{\prime} L_{\lambda \mathrm{i}} \cos \theta \mathrm{d} \Omega \mathrm{d} \lambda
$$

le symbole $\triangle$ signifiant que l'intégration sur $d \Omega$ prend en compte tout l'hémisphère entourant $\mathrm{d} S$. On en déduit sans difficulté les conditions radiatives à la surface du matériau, c'est-à-dire, par convention, le flux surfacique perdu $\varphi_{\mathrm{p}}$ :

$$
\varphi_{\mathrm{p}}=\varphi_{\mathrm{e}}-\varphi_{\mathrm{a}} .
$$

1.2 Facteurs totauX hémisphériques. CARACTÈre DÉCEVANT DES MESURES D'ABSORPTIVITÉ OU DE RÉFLECTIVITÉ. - Les bilans thermiques prenant en compte l'ensemble des directions de l'espace et toute l'échelle des longueurs d'onde, il peut sembler a priori plus avantageux de mesurer directement des facteurs totaux hémisphériques plutôt que de passer par l'intermédiaire de $\varepsilon_{\lambda}^{\prime}=\alpha_{\lambda}^{\prime}$ et de procéder aux doubles intégrations (9) et (10).

Pour une température $T$ superficielle donnée, l'émissivité totale hémisphérique $\varepsilon$ est une caractéris- 
tique du matériau considéré, compte tenu bien entendu de son état de surface; elle est reliée à $\varepsilon_{\lambda}^{\prime}$ par :

$$
\begin{array}{r}
\varepsilon(T)=\frac{\int_{\Delta} \int_{0}^{\infty} \varepsilon_{\lambda}^{\prime} L_{\lambda}^{0} \cos \theta \mathrm{d} \Omega \mathrm{d} \lambda}{\int_{0} \int_{0}^{\infty} L_{\lambda}^{0} \cos \theta \mathrm{d} \Omega \mathrm{d} \lambda}= \\
=\frac{\int_{\Delta} \int_{0}^{\infty} \varepsilon_{\lambda}^{\prime} L_{\lambda}^{0} \cos \theta \mathrm{d} \Omega \mathrm{d} \lambda}{\sigma T^{4}}
\end{array}
$$

$\sigma$ constante de Stefan-Boltzmann, $M^{0}=\sigma T^{4}$, émittance du corps noir. Le flux surfacique émis :

$$
\varphi_{\mathrm{e}}=\varepsilon \sigma T^{4}
$$

se calcule donc très aisément dès que les variations de $\varepsilon(T)$ en fonction du seul paramètre $T$ sont connues. Par ailleurs, le flux total hémisphérique émis par l'élément $\mathrm{d} S$ étant substantiellement plus élevé que celui $\mathrm{d} \phi_{\lambda e}$ rapporté à $\mathrm{d} \Omega$ et $\mathrm{d} \lambda$, il est possible de réaliser des mesures très précises de $\varepsilon(T)$, comme nous l'avons montré antérieurement [1].

Mais, contrairement à $\varepsilon(T)$, les absorptivités et réflectivités totales hémisphériques ne sont pas des caractéristiques du matériau puisqu'elles dépendent essentiellement du flux incident $d \phi_{\lambda i}$ qui varie totalement selon les conditions expérimentales ou pratiques d'emploi. C'est ainsi que l'absorptivité totale hémisphérique $\alpha_{i}$, définie par :

$$
\alpha_{\mathrm{i}}\left(T, L_{\lambda \mathrm{i}}\right)=\frac{\int_{0} \int_{0}^{\infty} \alpha_{\lambda}^{\prime} L_{\lambda \mathrm{i}} \cos \theta \mathrm{d} \Omega \mathrm{d} \lambda}{\int_{0} \int_{0}^{\infty} L_{\lambda \mathrm{i}} \cos \theta \mathrm{d} \Omega \mathrm{d} \lambda}
$$

ne peut être utilisée pour le calcul du flux surfacique absorbé :

$$
\varphi_{\mathrm{a}}=\alpha_{\mathrm{i}} \int_{0} \int_{0}^{\infty} L_{\lambda \mathrm{i}} \cos \theta \mathrm{d} \Omega \mathrm{d} \lambda=\alpha_{\mathrm{i}} \varphi_{\mathrm{i}}
$$

que si elle a été préalablement déterminée pour la même luminance $L_{\lambda \mathrm{i}}$ que celle intervenant dans le problème traité. Une telle obligation, très restrictive, est bien évidemment rarement réalisée et sa transgression conduit parfois à de graves erreurs.

Lorsqu'on s'intéresse à l'énergie solaire directe, le bilan thermique d'un capteur fait intervenir l'émissivité $\varepsilon(T)$ de l'absorbeur et son absorptivité totale directionnelle $\alpha_{S}^{\prime}$ :

$$
\alpha_{S}^{\prime}\left(T, \Delta, L_{\lambda S}\right)=\frac{\int_{0}^{\infty} \alpha_{\lambda}^{\prime} L_{\lambda S} \mathrm{~d} \lambda}{\int_{0}^{\infty} L_{\lambda S} \mathrm{~d} \lambda}
$$

fonction de la luminance monochromatique solaire, locale, instantanée, $L_{\lambda s}$ et de la direction $\Delta$ dans laquelle se trouve le soleil. Le flux surfacique absorbé (en l'absence de vitre) par l'absorbeur s'écrit :

$$
\varphi_{\mathrm{a}}=\alpha_{\mathrm{S}}^{\prime} \cos \theta_{\mathrm{S}} \Omega_{\mathrm{s}} \int_{0}^{\infty} L_{\lambda \mathrm{S}} \mathrm{d} \lambda
$$

$\theta_{\mathrm{S}}$ angle de $\Delta$ avec la normale au capteur, $\boldsymbol{\Omega}_{\mathrm{S}}$ angle solide sous lequel est vu le soleil. La température d'équilibre $T$ de l'absorbeur, obtenúe par égalisation de $\varphi_{\mathrm{a}}$ (17) et de $\varphi_{\mathrm{e}}$ (13), ne dépend que du rapport $\alpha_{S}^{\prime} / \varepsilon(T)$. De nombreux montages [1] permettent de mesurer $\alpha_{\mathrm{S}}^{\prime}$ ou le rapport $\alpha_{\mathrm{S}}^{\prime} / \varepsilon(T)$; mais, considérer ensuite ces deux grandeurs comme des caractéristiques de l'absorbeur, fonctions tout au plus de sa température, suppose que l'on néglige totalement les fluctuations de la luminance $L_{\lambda s}$ du rayonnement solaire direct. Par ailleurs, le flux absorbé par le même matériau à partir du rayonnement diffus ne peut pas être calculé à l'aide de $\alpha_{s}^{\prime}$; il fait intervenir en effet l'absorptivité hémisphérique totale $\alpha_{S}$ qui peut différer d'autant plus de $\alpha_{s}^{\prime}$ que $\alpha_{\lambda}^{\prime}$ évolue fortement avec $\Delta$ et que les compositions spectrales des rayonnements direct et diffus s'écartent l'une de l'autre.

Les considérations précédentes montrent que, si la détermination $\varepsilon(T)$ est intéressante, elle ne conduit qu'au flux émis $\varphi_{\mathrm{e}}$ mais ne donne aucune indication sur le flux absorbé $\varphi_{\mathrm{a}}$ et, par conséquent, ne suffit pas pour le calcul des échanges radiatifs à partir d'un matériau dont l'absorptivité $\alpha_{i}$ varie avec les conditions d'emploi.

\subsection{INTÉRÊT D'UNE MESURE MONOCHROMATIQUE} DIRECTIONNELLE. CHOIX DU FACTEUR SELON LE DOMAINE DE TEMPÉRATURe et DE LONGUEUR D'ONDE. - Par comparaison avec les difficultés évoquées ci-dessus, la détermination d'un facteur monochromatique directionnel présente un double intérêt :

- L'existence des relations (5), (6), (7) et (8) montre que l'obtention d'un seul facteur suffit; par exemple $\alpha_{\lambda}^{\prime}$ et $\rho_{\lambda \text { iso }}^{\prime \prime}=\rho_{\lambda}^{\prime}$ se déduisent de $\varepsilon_{\lambda}^{\prime}$. Il s'ensuit aussi qu'un facteur donné peut être atteint par voie directe ou indirecte, ce qui permet de choisir la plus précise ou, lorsque les deux voies sont utilisées conjointement, de procéder à des vérifications. De plus, il est possible d'accéder à des facteurs dont l'obtention directe serait quasi impossible; c'est le cas de l'absorptivité monochromatique directionnelle $\alpha_{\lambda}^{\prime}$, la quantité de chaleur absorbée à partir d'un flux incident très faible, puisque proportionnel à $\mathrm{d} \Omega$ et $\mathrm{d} \lambda$, n'étant guère décelable par voie calorimétrique.

- Dès qu'un facteur unique est connu, tout flux quel qu'il soit, émis, absorbé, réfléchi, se calcule immédiatement à partir d'expressions telles que (9) et (10), ce qui permet d'effectuer le bilan radiatif ou de traiter n'importe quel problème de transfert d'énergie radiative à condition d'adopter des lois de réflexion simples, spéculaire, isotrope ou association des deux. 
Le choix du facteur à mesurer n'est pas arbitraire; il dépend essentiellement du domaine de température et de longueur d'onde auquel on s'intéresse. $A$ priori, il est bien évidemment préférable de faire porter la mesure sur le facteur $\varepsilon_{\lambda}^{\prime}$ ou $\rho_{\lambda \text { iso }}^{\circ}=\rho_{\lambda}^{\prime \triangle}$ correspondant au flux le plus intense, que celui-ci soit émis ou réfléchi. Autrement dit (Fig. 2) :

- A basse température $T<500 \mathrm{~K}$, quel que soit $\lambda_{\text {, }}$ et à faible longueur d'onde, l'émission des matériaux est insuffisante pour être détectée et distinguée des réflexions parasites. Il faut faire appel à des mesures cie réflectivités.

- Dans le domaine $\lambda>2 \mu \mathrm{m}, T>500 \mathrm{~K}$, il est possible d'atteindre l'émissivité $\varepsilon_{\lambda}^{\prime}$. Mais, pour des matériaux très bons réflecteurs et à condition de disposer d'une source infrarouge suffisamment intense, une mesure de réflectivité peut s'avérer plus précise comme nous le montrerons plus loin.

D'après les considérations précédentes, on pourrait penser qu'il suffit de mettre au point un montage unique permettant d'atteindre $\rho_{\lambda \text { iso }}^{\prime \prime}$ ou $\rho_{\lambda}^{\prime \Delta}$, à condition toutefois de tolérer une incertitude relativement importante à haute température et grandes longueurs d'onde pour des matériaux très émissifs. En fait, la réalisation d'un tel montage, qui sera décrit dans le paragraphe 3, est assez délicate et il vaut mieux mesurer $\varepsilon_{\lambda}^{\prime}$ quand c'est possible. Par ailleurs, un certain nombre d'appareils (détecteurs, sources...) ou de revêtements (sphères intégrantes), adaptés à un domaine de longueur d'onde, le visible par exemple, ne le sont plus du tout dans un autre, comme l'infrarouge. Enfin, la plupart des mesures sont relatives, en ce sens qu'elles sont effectuées par référence à des caractéristiques radiatives supposées connues, qu'il s'agisse du flux émis par le corps noir $\mathrm{d} \phi_{\lambda}^{0}$ ou du flux réfléchi par une substance étalon (miroir, or...); une confrontation entre des résultats

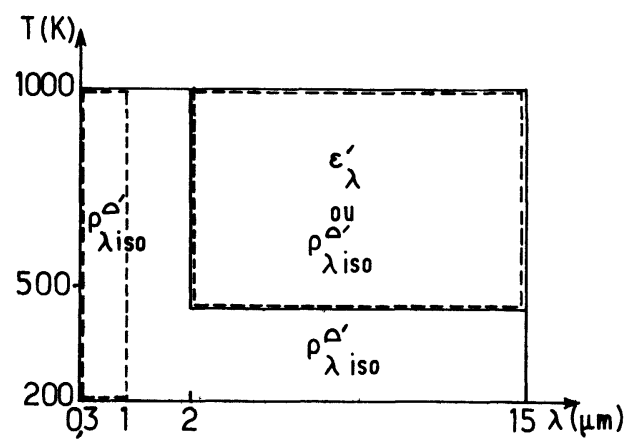

Fig. 2. - Choix du facteur radiatif à mesurer selon le domaine de température $T$ et de longueur d'onde $\lambda . \mathrm{La}$ mesure de $\rho_{\lambda \text { iso }}^{\prime}$ peut être remplacée par celle de $\rho_{\lambda}^{\prime}$. Les pointillés délimitent les zones actuellement accessibles à nos montages.

[Selection of the radiative factor to be measured according to the temperature $T$ and wavelength ranges $\lambda$. The measure of $\rho_{\lambda \text { iso }}^{\prime \prime}$ may be substituted by the measure of $\rho_{\lambda}^{\prime \circ}$. The dotted lines delimite the zone presently covered by our experimental set-ups.] d'origines différentes est alors souhaitable pour éviter toute erreur systématique.

Pour les raisons qui viennent d'être évoquées, deux types de montages destinés à mesurer, l'un $\varepsilon_{\hat{\lambda}}^{\prime}$, l'autre $\rho_{\lambda \text { iso, }}^{\circ \prime}$, sont utilisés conjointement dans nos laboratoires. Nous verrons qu'ils ne permettent pas de balayer le domaine présenté sur la figure $2: 0,3<\lambda<15 \mu \mathrm{m}$, $200<T<1000 \mathrm{~K}$; c'est pourquoi une troisième sorte de montage est en cours d'étude.

2. Mesures des émissivités monochromatiques directionnelles $\varepsilon_{\lambda}^{\prime}$. -2.1 MONTAGES RÉALISÉs. DOMAINE DE FONCTIONNEMENT : $2<\lambda<15 \mu \mathrm{m}$ ET $500<T<1000 \mathrm{~K}$. - Tous les montages concernant les émissivités $\varepsilon_{\lambda}^{\prime}$ comportent les mêmes éléments (voir référence [1]). Sans en reprendre la description générale, précisons certains de nos choix :

- L'utilisation d'un polariseur sur le trajet optique conduit à la détermination des émissivités $\varepsilon_{\lambda \|}^{\prime}$ et $\varepsilon_{\lambda \perp}^{\prime}$ correspondant aux deux directions habituelles de polarisation et donc à la moyenne $\varepsilon_{\lambda}^{\prime}=\frac{\varepsilon_{\lambda \|}^{\prime}+\varepsilon_{\lambda \perp}^{\prime}}{2}$ selon un calcul fait dans la référence [5].

- L'introduction d'un modulateur mécanique (ou hacheur), joint à une détection synchrone, élimine une bonne part des rayonnements parasites, mais laisse malheureusement subsister les flux réfléchis par l'échantillon; nous en verrons les conséquences dans le prochain paragraphe.

- Le contrôle des températures est assuré par deux régulateurs; l'un, absolu, maintient le corps noir à la température souhaitée; l'autre, différentiel, agit sur le système de chauffage de l'échantillon afin d'imposer à ce dernier la température du corps noir. Ce régulateur est attaqué par deux thermocouples identiques montés en différentiel et placés, l'un dans l'échantillon, l'autre au fond du corps noir. Malgré ces précautions, l'écart résiduel entre les deux températures reste l'une des principales causes d'erreur sur $\varepsilon_{\lambda}^{\prime}$ comme nous le verrons plus loin.

- La figure 3 montre un échantillon et son dispositif de chauffage; divers thermocouples, enfoncés jusqu'au niveau du milieu de l'échantillon, permettent d'en contrôler l'isothermie. La température superficielle $T$ est déterminée par la technique de l'extrapolation à partir des indications fournies par deux thermocouples placés à des distances connues de la surface. Cette technique donne des résultats très satisfaisants dans le cas d'échantillons bons conducteurs.

- Une augmentation de la sensibilité de la chaîne de mesure est obtenue par remplacement des détecteurs pyroélectriques par des détecteurs quantiques du type GeAu ou HgCdTe; plusieurs détecteurs sont . nécessaires pour couvrir la gamme $2-15 \mu \mathrm{m}$ avec tous les problèmes de réalignement optique que cela peut poser lors du changement en cours de manipulation.

- L'adoption de filtres interférentiels continûment variables à la place d'un monochromateur permet 


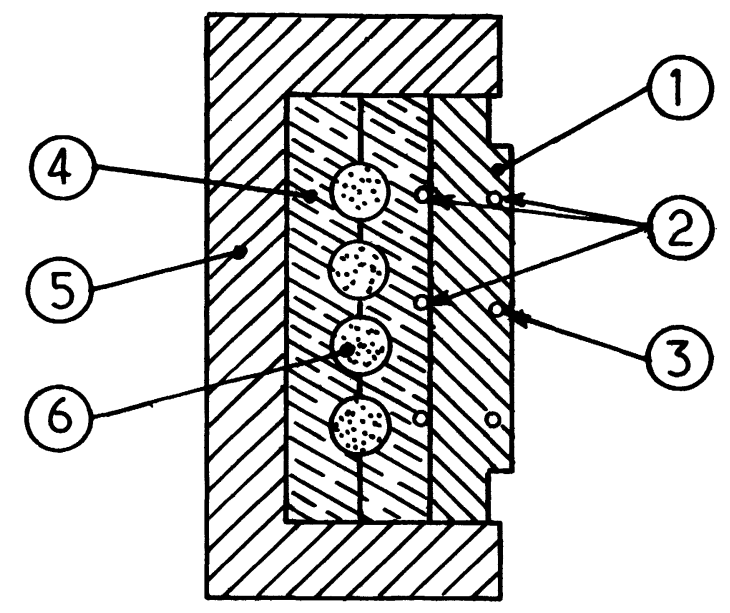

Fig. 3. - Porte échantillon avec le dispositif de chauffage et les thermocouples utilisés pour le repérage et la régulation de la température. (1) Echantillon, (2) Thermocouples intégrés, (3) Thermocouple branché sur le régulateur différentiel, (4) Cuivre, (5) Isolant, (6) Cartouche chauffante.

[Sample holder with the heating device and the thermocouples used for the measure and the regulation of temperature. (1) Sample, (2) Measurement thermocouples, (3) Thermocouple connected to the differential regulator, (4) Copper, (5) Insulator, (6) Heating resistor.]

d'aboutir à des montages plus compacts et de diminuer le nombre de miroirs.

- La mesure de l'émissivité totale directionnelle $\varepsilon^{\prime}$ est également possible; il suffit de supprimer le monochromateur et d'utiliser un détecteur dont la réponse spectrale, compte tenu des éléments de la chaîne optique, soit uniforme en fonction de $\lambda$. Cette condition n'est pas facilement réalisable mais, comparativement aux mesures de $\varepsilon_{\lambda}^{\prime}$, la précision sur $\varepsilon^{\prime}$ est favorisée par des flux radiatifs beaucoup plus importants.

- Enfin, pour éviter de mesurer successivement les flux émis par le corps noir et par l'échantillon, ces deux éléments étant placés à tour de rôle sur l'axe optique du montage, un autre progrès récent est l'adoption d'un système à double faisceau alterné qui évite les déplacements précédents [6].

Nous exploitons à l'heure actuelle trois appareillages basés sur le même principe mais de finalités différentes :

- Le premier [7, 8], mis en ouvre au Groupe de Recherches Thermiques, est destiné à l'étude de cavités de formes géométriques variées. L'échantillon comporte alors des sillons de sections droites régulières (dents de scie, créneaux, association des deux, ...); pour vérifier des résultats théoriques, établis à l'aide de la méthode de Monte Carlo par exemple $[9,10]$, il s'agit de mesurer une émissivité apparente $\varepsilon_{\lambda a}^{\prime}$ qui tient compte des réflexions successives à l'intérieur des cavités. Il est alors nécessaire de viser une surface suffisamment grande de l'échantillon pour inclure un nombre appréciable de sillons; cette surface visée doit par ailleurs être entièrement comprise dans l'ouverture du corps noir (modèle Pyrox). Le monochromateur est un spectromètre Perkin-Elmer. La chaîne optique est constituée par un miroir sphérique qui focalise le flux émis par l'échantillon (ou le corps noir) sur la fente d'entrée du spectromètre; un deuxième miroir sphérique renvoie l'image de la fente de sortie sur un détecteur $\mathrm{Ge}-\mathrm{Au}$.

- Si les échantillons étudiés ont une surface plane, lisse ou microrugueuse, l'obligation précédente, concernant l'étendue de la visée, n'existe plus. Mais l'influence sur $\varepsilon_{\lambda}^{\prime}$ de certains phénomènes tels que l'oxydation doit être d'autant plus examinée que l'on se propose d'élever la température $T$. Le corps noir du type IRCON (modèle BC 15) et l'échantillon sont alors placés dans une enceinte (Fig. 4) qui peut être entièrement vidée ou remplie d'un gaz donné. La chaîne optique comporte un seul miroir plan et une lentille $\mathrm{KBr}$. Le filtre continu dans l'infrarouge a un facteur de transmission de 40 à $60 \%$.

- Un dispositif réalisé à l'INSA de Lyon (Fig. 5) réunit en un seul montage la possibilité de mesurer $\varepsilon_{\lambda}^{\prime}$ et la réflectivité monochromatique bidirectionnelle $\rho_{\lambda}^{\prime \prime}[1]$. Dans ce dernier cas, la source est un globar et l'échantillon est placé sur le plateau d'un goniomètre Bouty; l'appareil fonctionne alors à température ordinaire et dans le domaine spectral 2-15 $\mu \mathrm{m}$ avec un détecteur de type pyroélectrique.

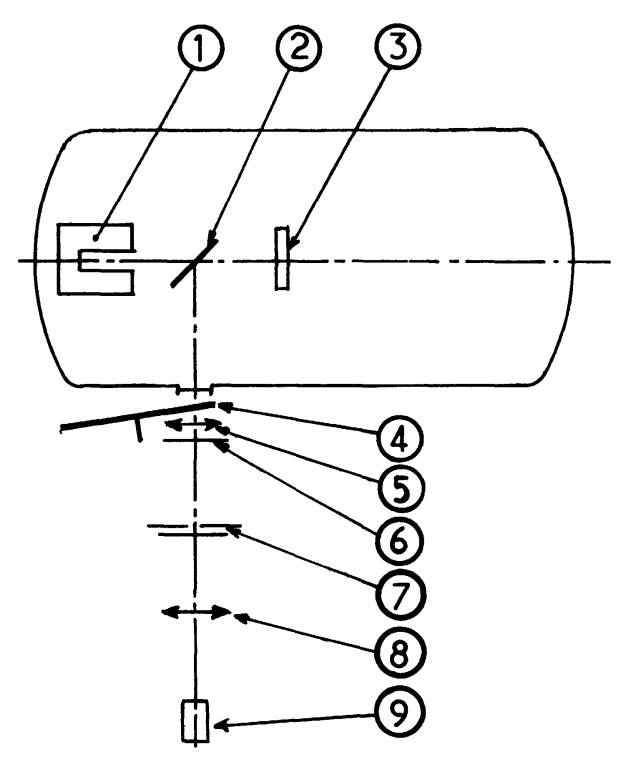

Fig. 4. - Schéma du montage de mesure de $\varepsilon_{\lambda}^{\prime}$ (Groupe de Recherches Thermiques). (1) Corps noir, (2) Miroir plan, (3) Echantillon, (4) Hacheur, (5) Lentille, (6) Polariseur infrarouge, (7) Filtre continu, (8) Lentille, (9) Détecteur.

[Sketch of the experimental set-up for the measure of $\varepsilon_{\lambda}^{\prime}$ (Groupe de Recherche Thermiques) : (1) Black body, (2) Plane mirror, (3) Sample, (4) Chopper, (5) Lens, (6) Infrared polarizer, (7) Continuous filter, (8) Lens, (9) Detector.] 


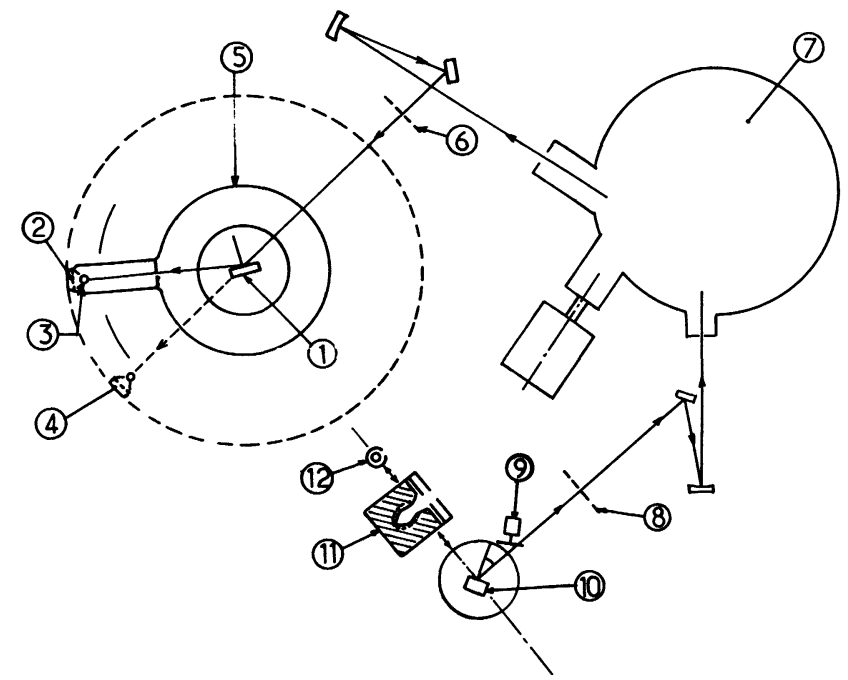

Fig. 5. - Schéma d'un dispositif de mesure de $\varepsilon_{\lambda}^{\prime}$ et de $\rho_{\lambda}^{\prime \prime}$ (INSA de Lyon). (1) Echantillon (mesure de $\rho_{\lambda}^{\prime \prime}$ ), (2) Miroir ellipsoïdal, (3) Détecteur (mesure de $\rho_{\lambda}^{\prime \prime}$ ), (4) Détecteur (mesure de $\varepsilon_{\lambda}^{\prime}$ ), (5) Goniomètre. (6) Polariseur (mesure de $\left.\rho_{\lambda}^{\prime \prime}\right)$, (7) Monochromateur à prisme $\mathrm{NaCl},(8)$ Polariseur (mesure de $\varepsilon_{\lambda}^{\prime}$ ), (9) Modulateur, (10) Echantillon (mesure de $\varepsilon_{\lambda}^{\prime}$ ), (11) Corps noir, (12) Globar.

[Sketch of the experimental set up for the measure of $\varepsilon_{\lambda}^{\prime}$ and $\rho_{\lambda}^{\prime \prime}$ (INSA Lyon) : (1) Sample (measure of $\rho_{\lambda}^{\prime \prime}$ ), (2) Ellipsoïdal mirror, (3) Detector (measure of $\rho_{\lambda}^{\prime \prime}$ ), (4) Detector (measure of $\varepsilon_{\lambda}^{\prime}$ ), (5) Goniometer, (6) Polarizer (measure of $\left.\rho_{\lambda}^{\prime \prime}\right),(7) \mathrm{NaCl}$ prism monochromator, (8) Polarizer (measure of $\varepsilon_{\lambda}^{\prime}$ ), (9) Chopper, (10) Sample (measure of $\varepsilon_{\lambda}^{\prime}$ ), (11) Black body, (12) Globular source.]

\subsection{Causes d'erreur. Précision des résultats. -} Certaines erreurs de mesures peuvent être évitées par des réglages préliminaires extrêmement soignés. C'est ainsi que l'axe optique de visée et l'axe de rotation de l'échantillon doivent être concourrants [11]. Un déca- lage éventuel introduit une erreur fonction de l'angle d'émission $\theta$; pour $\theta<80^{\circ}$, l'erreur est négligeable si la distance entre les deux axes ne dépasse pas $0,5 \mathrm{~mm}$. De même, avec des appareils convenables, tout défaut de polarisation peut être éliminé. Il reste à considérer deux causes d'erreurs essentielles [9] :

- La première provient d'une différence éventuelle de température $\Delta T$ entre le corps noir et l'échantillon. En admettant l'erreur relative ainsi introduite $\left(\Delta \varepsilon_{\lambda}^{\prime} / \varepsilon_{\lambda}^{\prime}\right)_{1}$, approximativement égale à la variation relative de luminance $\Delta L_{\lambda}^{0}(T) / L_{\lambda}^{0}(T)$ pour l'écart $\Delta T$, on trouve les résultats indiqués tableau 1. Comme on pouvait s'y attendre, $\left(\Delta \varepsilon_{\lambda}^{\prime} / \varepsilon_{\lambda}^{\prime}\right)_{1}$ décroît avec $T$ et $\lambda \mathrm{Si}$ les températures $T_{\mathrm{CN}}$ du corps noir et $T$ de l'échantillon sont réglées indépendamment l'une de l'autre, $\Delta T=\left|T_{\mathrm{CN}}-T\right|$ peut atteindre $5 \mathrm{~K}$ [7]. Grâce à l'utilisation des deux régulateurs mentionnée ci-dessus, $T$ ne dépasse pas $2 \mathrm{~K}$ [5].

- Certains flux réfléchis par l'échantillon s'associent au flux qu'il émet. A condition d'accorder une grande attention à la disposition de la chaîne de mesure, on peut éviter qu'une partie du flux partant de l'échantillon ne subisse des réflexions multiples entre ce dernier et le modulateur ou un élément du dispositif optique [9]; il suffit par exemple d'incliner légèrement la pale du modulateur par rapport à l'axe optique (Fig. 4) et de la réaliser avec un matériau comme l'aluminium à surface parfaitement polie et très réfléchissante. Mais une fraction du flux provenant de tout l'environnement (par exemple de l'enceinte) se réfléchit nécessairement sur l'échantillon. Si l'on suppose que ce milieu ambiant se comporte comme un corps noir à température $T_{\mathrm{a}}=300 \mathrm{~K}$, l'erreur relative $\left(\Delta \varepsilon_{\lambda}^{\prime} / \varepsilon_{\lambda}^{\prime}\right)_{2}$, voisine - de $\left[\left(1-\varepsilon_{\lambda}^{\prime}\right) / \varepsilon_{\lambda}^{\prime}\right]\left[L_{\lambda}^{0}\left(T_{\mathrm{a}}\right) / L_{\lambda}^{0}(T)\right]$, décroît, comme la précédente, en fonction de $T$, mais augmente avec $\lambda$; elle est par ailleurs d'autant plus grande que l'émissivité $\varepsilon_{\lambda}^{\prime}$ est faible; avec des émissivités supposées constantes : $\varepsilon_{\lambda}^{\prime}=0,3$ et $\varepsilon_{\lambda}^{\prime}=0,7$, on obtient les valeurs indiquées dans le tableau $\mathrm{I}$.

Tableau I. - Erreurs relatives (en \%) sur les mesures d'émissivités monochromatiques directionnelles $\varepsilon_{\lambda}^{\prime}$

\begin{tabular}{|c|c|c|c|c|c|c|c|c|c|c|}
\hline \multirow{2}{*}{\multicolumn{2}{|c|}{$T(\mathrm{~K})$}} & \multicolumn{3}{|c|}{$\begin{array}{c}600 \\
\lambda_{\mathrm{m}}(T)=5 \mu \mathrm{m}\end{array}$} & \multicolumn{3}{|c|}{ 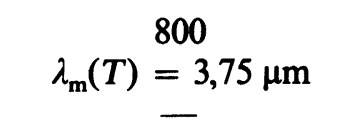 } & \multicolumn{3}{|c|}{$\begin{array}{c}1000 \\
\lambda_{\mathrm{m}}(T)=3 \mu \mathrm{m} \\
-\end{array}$} \\
\hline & & 2 & 5 & 10 & 2 & 5 & 10 & 2 & 5 & 10 \\
\hline$\left(\Delta \varepsilon_{\lambda}^{\prime}\right)$ & $\Delta T=5$ & 10 & 4 & 2 & 5,6 & 2,3 & 1,1 & 3,6 & 1,4 & 0,7 \\
\hline$\left(\overline{\varepsilon_{\lambda}^{\prime}}\right)_{1}$ & $\Delta T=2$ & 4 & 1,6 & 0,8 & 2,3 & 0,9 & 0,5 & 1,4 & 0,5 & néglig. \\
\hline$\left(\Delta \varepsilon_{\lambda}^{\prime}\right)$ & $\varepsilon_{\lambda}^{\prime}=0,3$ & néglig. & 2 & 21 & néglig. & 0,5 & 12 & néglig. & 0,3 & 7 \\
\hline$\left(\overline{\varepsilon_{\lambda}^{\prime}}\right)_{2}$ & $\varepsilon_{\lambda}^{\prime}=0,7$ & néglig. & 0,9 & 9 & néglig. & 0,2 & 5 & néglig. & 0,1 & 3 \\
\hline & $\Delta T=2$ & & & & & & & & & \\
\hline$\left(\Delta \varepsilon_{\lambda}^{\prime}\right)$ & $\varepsilon_{\lambda}^{\prime}=0,3$ & 4 & 3,6 & 22 & 2,3 & 1,4 & 12,5 & 1,4 & 0,8 & 7 \\
\hline$\left(\overline{\varepsilon_{\lambda}^{\prime}}\right)$ & $\varepsilon_{\lambda}^{\prime}=0,7$ & 4 & 2,5 & 9,8 & 2,3 & 1,1 & 5,5 & 1,4 & 0,6 & 3 \\
\hline
\end{tabular}


L'analyse précédente montre que l'erreur relative

$$
\frac{\Delta \varepsilon_{\lambda}^{\prime}}{\varepsilon_{\lambda}^{\prime}}=\left(\frac{\Delta \varepsilon_{\lambda}^{\prime}}{\varepsilon_{\lambda}^{\prime}}\right)_{1}+\left(\frac{\Delta \varepsilon_{\lambda}^{\prime}}{\varepsilon_{\lambda}^{\prime}}\right)_{2}
$$

décroît régulièrement avec $T$ mais passe par un minimum en fonction de $\lambda$. Autrement dit, à température relativement faible, les valeurs de $\varepsilon_{\lambda}^{\prime}$ ne sont suffisamment précises que pour une gamme restreinte de longueur d'onde; celle-ci s'élargit au fur et à mesure que $T$ croît et ceci d'autant plus que $\varepsilon_{\lambda}^{\prime}$ est grand. Un tableau tel que celui présenté ici montre les limites exactes du domaine valable, pour les mesures de $\varepsilon_{\lambda}^{\prime}$, mais la conclusion générale est évidente; dès que l'émission du matériau diminue, par suite d'une température $T$ trop basse, d'une longueur d'onde trop éloignée de celle correspondant au maximum $\lambda_{m}(T)$ de luminance du corps noir ou d'une faible valeur de $\varepsilon_{\lambda}^{\prime}$, il est préférable de recourir à des déterminations de réflectivités.

3. Mesures des réflectivités monochromatiques directionnelles $\rho_{\lambda}^{\prime \Delta}=\rho_{\lambda}^{\prime \prime}$ iso $^{\circ}$ - Comparativement aux mesures d'émissivités, celles basées sur la réflexion bénéficient $a$ priori de deux avantages :

- Le flux réfléchi est avant tout proportionnel à la puissance de la source, de sorte qu'il suffit en principe d'augmenter cette dernière pour pallier des faibles valeurs de réflectivités; de ce fait, la méthode peut être rendue relativement insensible aux variations de $\lambda$ et de $T$.

- La modulation du rayonnement incident, praticable dans certains montages, permet d'éliminer, grâce à la détection synchrone, le flux émis non modulé.

En contre partie, la mise au point de l'appareillage est plus difficile que pour l'émission. Avant de décrire le montage réalisé, il semble utile d'indiquer les raisons qui ont dicté certains de nos choix.

3.1 Difficultés COMPARATIVES DES MESURES DE $\rho_{\lambda}^{\prime \circ}$ ET DE $\rho_{\lambda \text { iso }}^{\prime}$ - Les principales difficultés, rencontrées dans la mesure de la réflectivité directionnelle hémisphérique $\rho_{\lambda}^{\prime \Delta}$, ont été mises en évidence dans le précédent article [1]; elles résident surtout dans la réalisation du dispositif destiné à recevoir l'ensemble du flux réfléchi, qu'il s'agisse de miroirs ou de sphères intégrantes. Il est quasiment impossible d'utiliser un détecteur à réponse angulaire uniforme et à surface suffisamment grande pour recueillir la totalité du rayonnement réfléchi par l'échantillon. De plus, les miroirs ou les revêtements de sphères n'ont pas une réflexion parfaite $\left(\rho_{\lambda}^{\prime},=1\right)$ pour toutes les longueurs d'onde et les directions, ce qui peut entraîner des erreurs importantes.

Si on examine les mesures antérieures de $\rho_{\lambda \text { iso }}^{\circ \prime}[1]$, ce sont les miroirs ou les sphères intégrantes qui sont chargés d'envoyer sur l'échantillon un flux rigoureusement isotrope; la réception du flux réfléchi directionnel par un détecteur ne pose alors aucun problème. Les réalisations se distinguent surtout par l'emplacement de la source. Celle-ci peut se trouver à l'intérieur du dispositif et éclairer directement le miroir ou la sphère; deux montages récents [12,13] utilisent ainsi une source annulaire entourant l'échantillon. Mais la modulation du rayonnement incident est quasiment impraticable alors qu'elle devient très aisée lorsque la source, placée à l'extérieur, éclaire miroir ou sphère par l'intermédiaire d'un diffuseur.

Un examen comparatif des différentes méthodes nous a conduit à préférer les mesures de $\rho_{\lambda \text { iso à celles de }}^{\text {' }}$ $\rho_{\lambda}^{\prime}$, essentiellement à cause des problèmes posés par le détecteur, et à éviter l'emploi de miroirs qui introduisent des réflexions parasites augmentant artificiellement la réflectivité de l'échantillon. Le recours à un montage de sphères intégrantes, utilisable pour une température $T$ relativement élevée de l'échantillon, implique l'option source à l'extérieur pour éliminer le flux émis, par modulation des flux incident et réfléchi; mais, comme nous allons le voir, l' isotropie de l'éclairement de l'échantillon est alors plus difficile à assurer.

3.2 MONTAGE RÉALISÉ POUR MESURER $\rho_{\lambda \text { iso ENTRE }}^{\prime}$ 0,3 ET $1 \mu \mathrm{m}$. - La mise en cuvre d'une sphère intégrante est basée sur la réalisation d'un revêtement très réfléchissant, afin d'obtenir un rendement correct (le flux détecté ne doit pas être trop petit par rapport au flux entrant dans la sphère [11]), et également très diffusant, pour que les réflexions multiples produisent un éclairement uniforme de la sphère. Ces conditions sont facilement satisfaites dans le visible, soit par dépôt d'une couche épaisse ( $\simeq 2 \mathrm{~mm}$ ) d'oxyde de magnésium, soit par l'emploi de diverses peintures. Dans l'infrarouge, les revêtements, constitués par de petites billes de verre collées sur la paroi et recouvertes successivement de chrome et d'or, se comportent correctement avec une source placée à l'intérieur [12]; mais dans le cas contraire (source à l'extérieur), des essais préliminaires ne nous ont pas donné satisfaction ; conformément à des observations antérieures [14], l'anisotropie du flux reçu par l'échantillon interdisait toute mesure pour des angles dépassant $40^{\circ}$ environ. Nous avons donc opté pour un revêtement réalisé par dépôt d'une dizaine de couches de peinture au sulfate de baryum dont nous avons contrôlé les performances de 0,3 à $1 \mu \mathrm{m}[11]$.

$\mathrm{Si}$ on considère la sphère intégrante, au centre de laquelle doit se trouver l'échantillon pourvu de systèmes de rotation et de chauffage (Fig. 6), il est impossible d'imposer un éclairement uniforme sur toute la surface de la sphère par suite des ombres portées; mais on peut remarquer que ce n'est pas nécessaire. En effet, pour connaître la dépendance de $\rho_{\lambda \text { iso }}^{\text {' en }}$ fonction de $\theta$, il suffit de faire tourner l'échantillon d'un angle $\theta$ variant de 0 à $90^{\circ}$ autour d'un axe situé sur sa surface et perpendiculaire à la direction $\Delta$ d'observation; il est alors facile de constater qu'au cours de sa rotation, l'échantillon ne « voit » que les trois quarts de la surface interne de la sphère. 


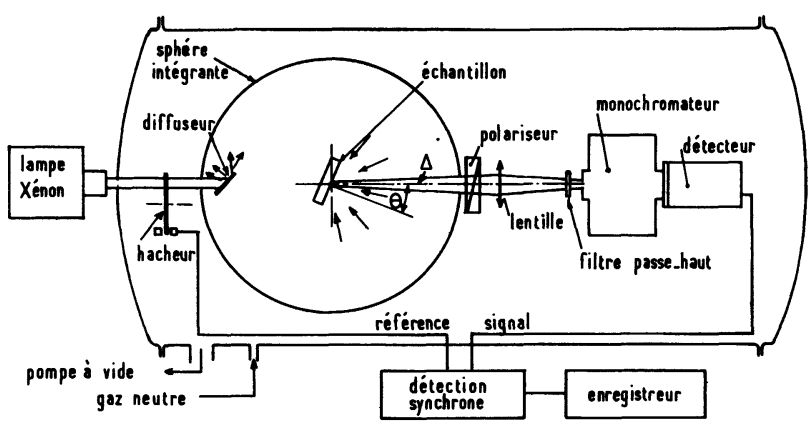

Fig. 6. - Schéma du montage de mesure de $\rho_{\lambda \text { iso }}^{\prime \prime}$ (Groupe de Recherches Thermiques).

[Sketch of the experimental set up for the measure of $\rho_{\lambda \text { iso }}^{\prime}$ (Groupe de Recherches Thermiques).]

Des expériences préliminaires aussi bien que des calculs [11] nous ont amenés à la conception du dispositif schématisé sur la figure 6 , contenu dans une enceinte sous atmosphère contrôlée et exploité au Groupe de Recherches Thermiques, en premier lieu pour $200<T<500 \mathrm{~K}$ et dans les conditions suivantes :

- La source, modulée par un hâcheur mécanique, éclaire, près de l'orifice d'entrée, un petit disque, placé à l'intérieur de la sphère, recouvert par le même revêtement et tourné de manière à éclairer par réflexion un quart de sphère. Les trois quarts utiles ne sont alors éclairés qu’à partir de réflexions multiples, assurant une uniformité et une isotropie parfaites au rayonnement qu'ils diffusent.

- Le contrôle de la température de l'échantillon est assuré par un flux d'air, régulé en température et débit, traversant le porte échantillon creux.

- Le spectre de la lampe à xénon, qui s'étend de 0,25 à $1,5 \mu \mathrm{m}$, est pratiquement plat entre 0,4 et $0,8 \mu \mathrm{m}$, mais présente des pics importants dans le proche infrarouge; la densité spectrale est de l'ordre de $5 \mathrm{~W} \cdot \mu \mathrm{m}^{-1}$ dans le visible et les variations d'intensité ne dépassent pas 1 à $2 \%$.

- Les mesures peuvent être relatives ou absolues. Dans le premier cas, la référence est constituée d'acier inoxydable $304 \mathrm{~L}$ poli, sur lequel est déposé un film d'aluminium protégé par une couche de silice; sa réflectivité monochromatique directionnelle, correspondant à une réflexion spéculaire, a été mesurée à l'aide de deux méthodes différentes, par J. Lafait, au Laboratoire d'Optique des Solides de Paris VI, et par nous-mêmes à l'aide d'un laser He-Ne [11]. Pour des mesures absolues, une première détermination est effectuée en l'absence d'échantillon, le signal étant alors proportionnel au flux réfléchi par la sphère intégrante; il est à remarquer que la réflexion par le revêtement doit être isotrope, mais"peut ne pas être parfaite, au prix toutefois d'une perte de rendement.

Les montages réalisés récemment bénéficient de plusieurs améliorations. Dans nos deux laboratoires, les orifices d'entrée et de sortie du rayonnement ne sont plus placés sur le même diamètre (Fig. 7). La sphère utilisée au Groupe de Recherches Thermiques possède désormais une double enveloppe refroidie par une circulation d'eau, ce qui permet d'élever la température de l'échantillon jusqu'à environ $1000 \mathrm{~K}$ et, corrélativement, de protéger la peinture au sulfate de Baryum dont la réflectivité s'altère au-delà de $350 \mathrm{~K}$; par ailleurs, le monochromateur à réseau a été remplacé par un jeu de filtres sélectifs.

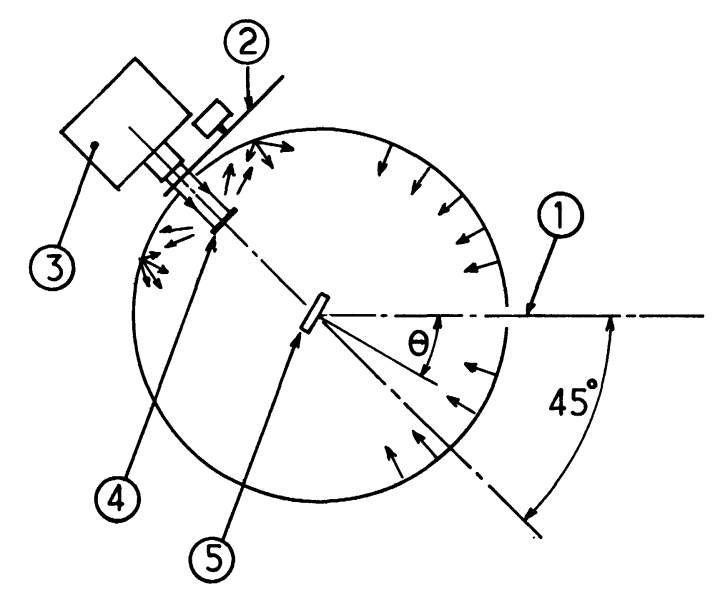

Fig. 7. - Nouvelle disposition de la sphère intégrante. (1) Axe de mesure, (2) Modulateur, (3) Source, (4) Diffuseur, (5) Echantillon.

[New arrangement of the integrating sphere. (1) Measure axis, (2) Chopper, (3) Xenon lamp, (4) Diffuser, (5) Sample.]

3. 3 Causes d'erreur. Précision des résultats. Comme pour les mesures d'émissivité, nous supposerons que certaines erreurs sont rendues négligeables par des précautions préliminaires : défauts d'alignement et de polarisation par exemple. Les flux parasites sont ici totalement éliminés. On peut estimer à $2 \%$ environ [11] l'incertitude sur $\rho_{\lambda \text { iso }}^{\mathrm{\prime}}$, introduite par les variations d'intensité de la source et par les mesures du flux réfléchi par la référence ou par la sphère.

Il reste à examiner la principale cause d'erreur due aux imperfections de la luminance du revêtement qui n'est, en toute rigueur, ni uniforme ni isotrope; en dehors du diffuseur et de l'échantillon lui-même, des perturbations sont introduites par la présence de l'orifice de mesure. Les erreurs ainsi commises, difficiles à chiffrer du reste, dépendent en plus de la nature du matériau par le biais des lois de réflexion. A l'aide de la référence utilisée comme un miroir, nous avons donc mesuré les variations du flux incident sur l'échantillon en fonction de sa position, c'est-à-dire de l'angle $\theta$, et calculé l'erreur relative $\left(\Delta \rho_{\lambda}^{\prime}{ }_{\text {iso }}^{\prime} / \rho_{\lambda}^{\prime}{ }_{\text {iso }}^{\prime}\right)_{1}$ dans le cas d'une réflexion, soit spéculaire, soit diffuse isotrope [11]. Le tableau II indique les valeurs ainsi obtenues et l'erreur relative totale, compte tenu de l'incertitude de $2 \%$ signalée 
ci-dessus. $\Delta \rho_{\lambda \text { iso }}^{\mathrm{\prime}} / \rho_{\lambda \text { iso }}^{\mathrm{\partial}}$ est pratiquement indépendant de $T$ et ne varie pas en fonction de $\lambda$ pour

$$
0,3<\lambda<1 \mu \mathrm{m} \text {. }
$$

Tableau II. - Erreurs relatives (en \%) sur les mesures de réflectivités monochromatiques directionnelles.

\begin{tabular}{|c|c|c|c|c|}
\hline \multirow{2}{*}{$\begin{array}{l}\text { Echantillon } \\
\text { à réflexion } \\
-\end{array}$} & \multicolumn{2}{|c|}{ Spéculaire } & \multicolumn{2}{|c|}{$\begin{array}{c}\text { Diffuse } \\
\text { isotrope }\end{array}$} \\
\hline & - & - & - & - \\
\hline Angle $\theta$ & 10 & 80 & 10 & 80 \\
\hline$\left(\frac{\Delta \rho_{\lambda \text { iso }}^{\Delta \prime}}{\rho_{\lambda \text { iso }}^{\prime \prime}}\right)_{1}^{\prime \prime}$ & négligeable & 2,5 & 3 & 2,5 \\
\hline$\frac{\Delta \rho_{\lambda \text { iso }}^{\circ}}{\rho_{\lambda \text { iso }}^{\prime \prime}}$ & 2 & 4,5 & 5 & 4,5 \\
\hline
\end{tabular}

Une comparaison des précisions sur $\varepsilon_{\lambda}^{\prime}$ (tableau I) et $\rho_{\lambda \text { iso }}^{\prime \prime}$ (tableau II) est difficile puisque les domaines de longueur d'onde diffèrent. Mais elle confirme la complémentarité, prévue a priori, des deux types de montage; les déterminations de $\rho_{\lambda \text { iso deviennent }}^{\partial \prime}$ préférables à celles de $\varepsilon_{\lambda}^{\prime}$ par exemple à basse température ou à grande longueur d'onde.

4. Contrôle de la fiabilité des montages. - Pour un même échantillon et dans des conditions identiques de température, de longueur d'onde et de direction $\Delta$, les résultats de nos mesures d'émissivité $\varepsilon_{\lambda}^{\prime}$ et de réflectivité $\rho_{\lambda \text { iso }}^{\alpha \prime}$ ont été plusieurs fois (et sont encore) comparés avec ceux provenant de montages différents, mis au point dans d'autres Laboratoires universitaires ou industriels. Il n'est évidemment pas question de présenter ici toutes ces confrontations; nous nous limiterons à un exemple un peu particulier puisqu'il ne satisfait pas au critère «longueur d'onde identique».

$\mathrm{La}$ réalisation d'une "bonne" sphère intégrante n'étant pas très facile, nous avons tenu en premier lieu à contrôler la fiabilité de notre dispositif, en mesurant conjointement la réflectivité $\rho_{\lambda \text { iso }}^{\circ}$ par notre méthode et à l'aide d'un montage, basé sur un autre principe et dépourvu de sphère. Pour un échantillon à réflexion spéculaire, un tel montage a été mis au point [1] au Laboratoire d'Optique des Solides de Paris VI; il ne fonctionne toutefois qu'à la température ordinaire et pour $\lambda>2 \mu \mathrm{m}$. Nous avons donc été amenés à comparer des mesures effectuées dans des domaines différents de longueur d'onde $[15,16]$.

La figure 8 représente, pour une incidence normale $\theta=0$, l'émissivité monochromatique directionnelle $\varepsilon_{\lambda}^{\prime}(\theta=0)$ de l'acier inoxydable $304 \mathrm{~L}$, à surface parfaitement polie (donc à réflexion spéculaire) et à la température $T=300 \mathrm{~K}$. Les expériences ont porté sur un échantillon, d'abord non oxydé, puis oxydé par chauffage préalable à différentes températures. Comme le montrent les différentes courbes tracées, les points, obtenus à courtes longueurs d'onde à partir de $\varepsilon_{\lambda}^{\prime}(\theta=0)=1-\rho_{\lambda}^{\prime}{ }_{\text {iso }}^{\prime}(\theta=0)$, se raccordent parfaitement à ceux trouvés dans l'infrarouge.

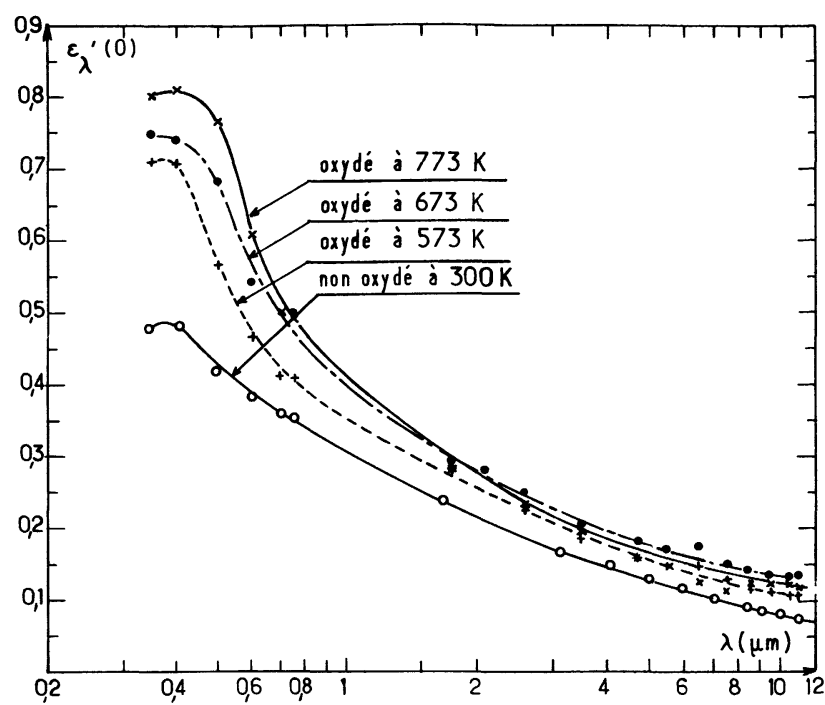

Fig. 8. - Emissivité monochromatique normale $\varepsilon_{\lambda}^{\prime}(0)$ mesurée à $300 \mathrm{~K}$ pour l'acier $304 \mathrm{~L}$ à surface parfaitement polie, non oxydée, puis oxydée préalablement à $573 \mathrm{~K}$ $(+-+-+), 673 \mathrm{~K}(-\cdot-\cdot-), 773 \mathrm{~K}(\times-x-\times)$.

[Normal monochromatic emissivity $\varepsilon_{\lambda}^{\prime}(0)$ of burnished stainless Steel $304 \mathrm{~L}$ measured at $300 \mathrm{~K}$. unoxidized, +-+-+ previously oxidized at $573 \mathrm{~K}, \cdot-\cdot-\cdot-$ previously oxidized at $673 \mathrm{~K}, x-x-x-$ previously oxidized at $773 \mathrm{~K}$.]
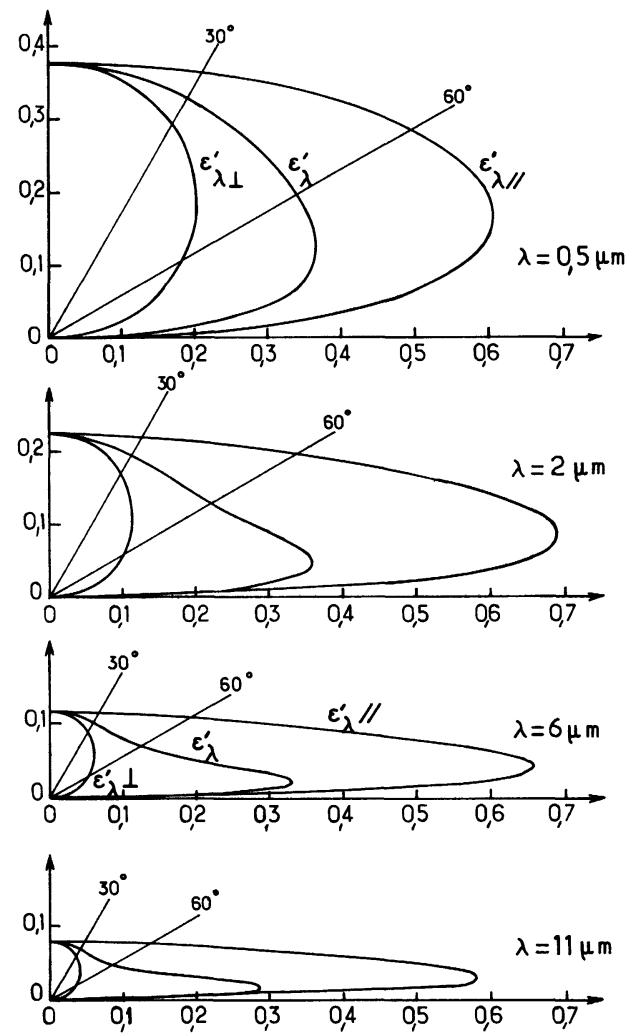

Fig. 9. - Indicatrices d'émissivité monochromatique $\varepsilon_{\lambda}^{\prime}(\theta)$ de l'acier inoxydable $304 \mathrm{~L}$ non oxydé, à surface parfaitement polie et porté à une température de $300 \mathrm{~K}$, pour différentes longueurs d'onde.

[Monochromatic emissivity $\varepsilon_{\lambda}^{\prime}(\theta)$ as a function of the angle of emission for unoxidized burnished stainless steel $304 \mathrm{~L}$ at $300 \mathrm{~K}$ for different wavelengths.] 
La même concordance s'observe si l'on envisage les variations de $\varepsilon_{\lambda}^{\prime}$ en fonction de l'angle $\theta$, pour différentes longueurs d'onde. Les indicatrices d'émissivité de l'échantillon d'acier inox $304 \mathrm{~L}$ non oxydé (Fig. 9) se déforment régulièrement lorsque $\lambda$ croît, sans qu'il existe de discontinuités entre 0,5 et $2 \mu \mathrm{m}$; ceci peut être confirmé par le tracé de courbes identiques à celles de la figure 8 , mais correspondant à $\theta$ différent de 0 .

Les domaines d'exploitation de nos montages ne se recouvrent pas; comme l'illustre la figure 8 , nous ne pouvons obtenir aucun résultat entre 1 et $2 \mu \mathrm{m}$, quelle que soit $T$, et, à basse température, pour $\lambda>2 \mu \mathrm{m}$. Il s'agit là d'un phénomène général si on se réfère au nombre très insuffisant de données dans ces deux zones, fort intéressantes cependant sur le plan pratique. En effet, pour les substances sélectives utilisées dans les capteurs solaires, c'est au voisinage de $1 \mu \mathrm{m}$ que se situent les variations importantes de $\varepsilon_{\lambda}^{\prime}$; par ailleurs, en ce qui concerne le bâtiment par exemple, il est indispensable de connaître l'émission de matériaux à réflexion diffuse, dans l'infrarouge, à température ordinaire. Les lacunes que nous venons de signaler doivent donc être comblées; il faut pouvoir déterminer $\varepsilon_{\lambda}^{\prime}$ ou $\rho_{\lambda \text { iso }}^{\prime \prime}$ dans tout le domaine schématisé figure 2 .

5. Conclusion. - Par suite de l'inévitable faiblessè des flux détectés, les mesures radiatives monochromatiques directionnelles exigent la mise au point de montages particulièrement soignés et performants. Leur exactitude et leur précision sont subordonnées néanmoins à des aléas difficilement maîtrisables : instabilité d'intensité des sources, utilisation de corps noirs ou de références plus ou moins valables, dérives dans les régulations de température... De par sa définition même, l'émissivité $\varepsilon_{\lambda}^{\prime}$ ne peut être atteinte directement qu'à température $T$ élevée et à grande longueur d'onde $\lambda$ Si une telle restriction ne s'applique pas à la réflectivité $\rho_{\lambda \text { iso, certaines }}^{\prime \prime}$ contingences pratiques telles que les qualités de diffusion d'un revêtement, la sensibilité des détecteurs, l'existence de sources suffisamment puissantes sans être trop onéreuses... limitent le fonctionnement de n'importe quel système à des bandes relativement étroites de $\lambda$ et de $T$. Malgré les deux montages dont nous disposons au Laboratoire, certaines zones nous restent inaccessibles. Notre but actuel est donc l'élaboration d'un dispositif permettant non seulement de recouvrir tout le domaine $0,3<\lambda<15 \mu \mathrm{m}$, $200<T<1000 \mathrm{~K}$, mais aussi d'étendre substantiellement les mesures de $\rho_{\lambda \text { iso }}^{\text {' }}$ afin de les substituer à celles de $\varepsilon_{\lambda}^{\prime}$ quand ces dernières manquent de précision; un concentrateur spécial permettant de faire rentrer un flux plus important dans la sphère est en cours d'étude.

Remerciements. - Les auteurs souhaitent remercier MM. Abeles et Lafait pour leurs conseils et pour l'aide que leur a apportée le Laboratoire d'Optique des Solides de l'Université Pierre et Marie Curie. Ils tiennent à souligner la participation importante de MM. J. M. Ané, P. Demont, P. Stekelorom, H. Tran N'Guyen aux travaux exposés dans cet article.

\section{Bibliographie}

[1] Demont, P., Huetz-Aubert, M., Sacadura, J. F., Revue Phys. Appl. 17 (1982) 239.

[2] Siegel, R., Howell, J. R., Thermal Radiation Heat Transfer (McGraw Hill Book, Company, New York) 1972.

[3] Huetz-Aubert, M., TAine, J., Rev. Gén. Thermique XVII (1978) 755.

[4] Born, M., Wolf, E., Principles of optics (Pergamon Press, Oxford) 1975.

[5] Sacadura, J. F., Thèse Doctorat d'Etat, Lyon I, juillet 1980.

[6] Sacadura, J. F., Quinto Diez, P., Heinisch, B., VIIe Congrès International sur les Transferts de Chaleur, Munich, 1982.

[7] Druelle, Ph., Thèse Docteur Ingénieur, Ecole Centrale des Arts et Manufactures, Paris, octobre 1978.

[8] Druelle, Ph., Huetz-Aubert, M., VIe Congrès International sur les Transferts de Chaleur, Toronto, août 1978.
[9] Tran N'Guyen, H., Thèse docteur Ingénieur, Ecole Centrale des Arts et Manufactures, Paris, juin 1981.

[10] Demont, Ph., Tran N'Guyen, H., Comportement Radiatif des Surfaces Rugueuses, Groupement Universitaire de Thermique, mai 1979.

[11] Demont, Ph., Thèse Docteur Ingénieur, Ecole Centrale des Arts et Manufactures, Paris, novembre 1980.

[12] Imbert, C., Thèse Docteur Ingénieur, Paris XI, 1969.

[13] Behaghel, J. M., Thèse Docteur Ingénieur, Paris VI, 1980.

[14] Godon, J. C., Thèse 3e cycle, Paris VI, 1977.

[15] Demont, Ph., Tran N'Guyen, H., Sacadura, J. F., Conférence Internationale sur les Matériaux pour la Conversion Photothermique de l'Energie Solaire, Ajaccio, mai 1980, J. Physique Colloq. 42 (1981) C1-161.

[16] Ane, J. M., Sacadura, J. F., Stekelorom, P., Vile Congrès International sur les Transferts de Chaleur, Munich, 1982. 